\title{
Nonlinear Neuromuscular Electrical Stimulation Tracking Control of a Human Limb
}

\author{
Nitin Sharma, Keith Stegath, Chris M. Gregory, and Warren E. Dixon
}

\begin{abstract}
A high-level objective of neuromuscular electrical stimulation (NMES) is to enable a person to achieve some functional task. Towards this goal, the objective of the current effort is to develop a NMES controller to produce a knee position trajectory that will enable a human shank to track any continuous desired trajectory (or constant setpoint). A nonlinear control method is developed to control the human quadriceps femoris muscle undergoing nonisometric contractions. The developed controller does not require a muscle model and can be proven to yield asymptotic stability for a nonlinear muscle model in the presence of bounded nonlinear disturbances (e.g., spasticity, delays, fatigue). The performance of the controller is demonstrated through a series of closed-loop experiments on human subjects. The experiments illustrate the ability of the controller to enable the leg shank to track single and multiple period trajectories with different periods and ranges of motion, and also track desired step changes with changing loads.
\end{abstract}

Index Terms-Asymptotic stability, Lyapunov methods, neuromuscular electrical stimulation (NMES), nonlinear control, RISE feedback.

\section{INTRODUCTION}

$\mathbf{N}$ EUROMUSCULAR electrical stimulation (NMES) is the application of a potential field across a muscle via internally or externally placed electrodes in order to produce a desired muscle contraction. NMES is a prescribed treatment for a number of neurological dysfunctions. Because of the potential for improvements in daily activities by people with movement disorders such as stroke and spinal cord injuries, the development of NMES as a neuroprosthesis has grown rapidly [1]. However, the application and growth of NMES technologies have been stymied by several technical challenges related to the design of an automatic stimulation strategy. Specifically, due to a variety of uncertainties in muscle physiology (e.g., temperature, $\mathrm{pH}$, and architecture), predicting the exact contraction force exerted by the muscle is difficult. One cause of this difficulty is that there is an unknown mapping between the generated

Manuscript received June 27, 2008; revised January 20, 2009; accepted March 03, 2009. First published June 02, 2009; current version published December 16, 2009. This work was supported by the National Science Foundation under CAREER Award CMS-0547448.

N. Sharma, K. Stegath, and W. E. Dixon are with the Department of Mechanical and Aerospace Engineering, University of Florida, Gainesville, FL 32611 USA (e-mail: kstegath@ufl.edu; robero@ufl.edu; wdixon@ufl.edu).

C. M. Gregory is with the Brain Rehabilitation Research Center, North Florida/South Georgia Veterans Health System Department of Physical Therapy, University of Florida, Gainesville, FL 32611 USA (e-mail: cgregory@phhp.ufl.edu).

Color versions of one or more of the figures in this paper are available online at http://ieeexplore.ieee.org.

Digital Object Identifier 10.1109/TNSRE.2009.2023294 muscle force and stimulation parameters. There are additional problems with delivering consistent stimulation energy to the muscle due to a variety of factors including: electrode placement, percentage of subcutaneous body fat, muscle fatigue, as well as overall body hydration.

Given the uncertainties in the structure of the muscle model and the parametric uncertainty for specific muscles, some investigators have explored various linear PID-based pure feedback methods (cf. [2]-[5] and the references within). Typically, these approaches have only been empirically investigated and no analytical stability analysis has been developed that provides an indication of the performance, robustness, or stability of these control methods. Some recent studies (e.g., see [6]) also point to evidence that suggests that linear control methods do not yield acceptable performance in practice. The development of a stability analysis for previous PID-based NMES controllers has been evasive because of the fact that the governing equations for a muscle contraction/limb motion are nonlinear with unstructured uncertainties. Some efforts have focused on analytical control development for linear controllers (e.g., [4], [7], [8]); however, the governing equations are typically linearized to accommodate a gain scheduling or linear optimal controller approach.

Motivated by the lack of control development for PID-based feedback methods, significant research efforts have focused on the use of neural network-based controllers (cf. [9]-[13] and the references within). Nonlinear neural network methods provide a framework that allows the performance, robustness, and stability of the developed NMES controllers to be investigated without linearization assumptions. However, all of the previous neural network-based NMES controllers are limited to a uniformly ultimately bounded result because of the inevitable residual nonlinear function approximation error. Additionally, neural networks may exhibit performance degradation during the transient phase while the estimates update.

An open-loop error system for a general uncertain nonlinear muscle model is developed in this paper (and in the preliminary results in [14]) by grouping terms in a manner that facilitates the development of a new continuous feedback method (coined RISE for robust integral of the sign of the error in [15], [16]). Through this error-system development, the continuous RISE controller is proven (through a Lyapunov-based stability analysis) to yield an asymptotic stability result despite the uncertain nonlinear muscle model and the presence of additive bounded disturbances (e.g., muscle spasticity, fatigue, changing loads in functional tasks, and delays). The performance of the nonlinear controller is experimentally verified for tracking of a human leg shank by applying the controller as a voltage potential across external electrodes attached to the distal-medial and 

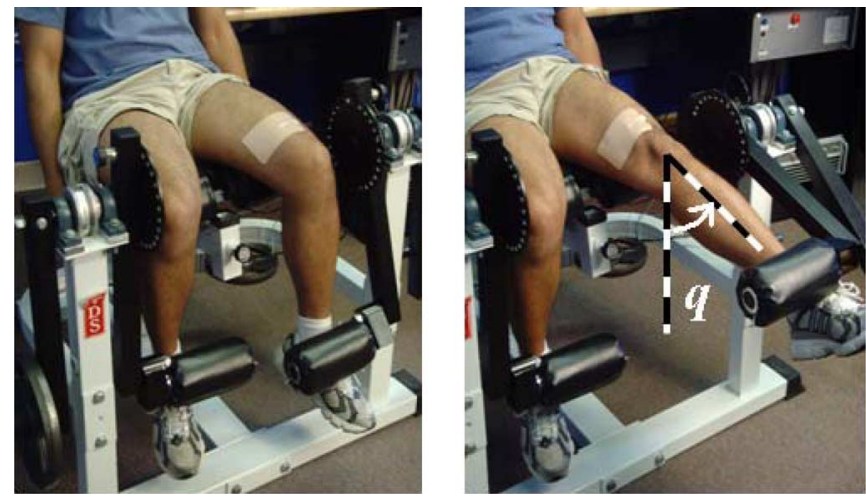

Fig. 1. In left image the subject's left leg is in relaxed state. The right image shows the left leg during stimulation. The angle $\mathrm{q}(\mathrm{t})$ is measured with respect to the vertical line as shown.

proximal-lateral portion of the quadriceps femoris muscle group. The RISE controller is implemented by a voltage modulation scheme with a fixed frequency and a fixed pulse width. Other modulation strategies (e.g., frequency or pulse-width modulation) could have also been implemented (and applied to other skeletal muscle groups) without loss of generality. The experiments illustrate the ability of the controller to enable the leg shank to track single and multiple period trajectories with different periods and ranges of motion, and also track desired step changes with changing loads. Future research will focus on including physiological muscle dynamics in the control structure through adaptive feedforward terms.

\section{Muscle Activation AND Limb Model}

The total muscle knee joint model can be categorized into body segmental dynamics and muscle activation and contraction dynamics. The muscle activation and contraction dynamics explains the force generation in the muscle while the body segmental dynamics considers the active moment and passive joint moments.

The total knee-joint dynamics can be modeled as [4]

$$
M_{I}+M_{e}+M_{g}+M_{v}+\tau_{d}=\tau .
$$

In (1), $M_{I}(\ddot{q}) \in \mathbb{R}$ denotes the inertial effects of the shank-foot complex about the knee-joint, $M_{e}(q) \in \mathbb{R}$ denotes the elastic effects due to joint stiffness, $M_{g}(q) \in \mathbb{R}$ denotes the gravitational component, $M_{v}(\dot{q}) \in \mathbb{R}$ denotes the viscous effects due to damping in the musculotendon complex [17], $\tau_{d}(t) \in \mathbb{R}$ is considered as an unknown bounded disturbance which represents an unmodeled reflex activation of the muscle (e.g., muscle spasticity) and other unknown unmodelled phenomena (e.g., dynamic fatigue, electromechanical delays), and $\tau(t) \in \mathbb{R}$ denotes the torque produced at the knee joint. In the subsequent development, the unknown disturbance $\tau_{d}(t)$ is assumed to be bounded and its first and second time derivatives are assumed to exist and be bounded. These are reasonable assumptions for typical disturbances such as muscle spasticity, fatigue, and load changes during functional tasks. For simplicity, the passive damping and elastic force of muscle and joints are considered together.
The inertial and gravitational effects in (1) can be modelled as

$$
M_{I}(\ddot{q}(t))=J \ddot{q}(t), \quad M_{g}(q(t))=-m g l \sin (q(t))
$$

where $q(t), \dot{q}(t), \ddot{q}(t) \in \mathbb{R}$ denote the angular position, velocity, and acceleration of the lower shank about the knee-joint, respectively (see Fig. 1), $J \in \mathbb{R}$ denotes the unknown inertia of the combined shank and foot, $m \in \mathbb{R}$ denotes the unknown combined mass of the shank and foot, $l \in \mathbb{R}$ is the unknown distance between the knee-joint and the lumped center of mass of the shank and foot, and $g \in \mathbb{R}$ denotes the gravitational acceleration. The elastic effects are modelled on the empirical findings by Ferrarin and Pedotti in [17] as

$$
M_{e}(q)=-k_{1}\left(\exp \left(-k_{2} q(t)\right)\right)\left(q(t)-k_{3}\right)
$$

where $k_{1}, k_{2}, k_{3} \in \mathbb{R}$ are unknown positive coefficients. As shown in [4], the viscous moment $M_{v}(\dot{q})$ can be modelled as

$$
M_{v}(\dot{q}(t))=B_{1} \tanh \left(-B_{2} \dot{q}(t)\right)-B_{3} \dot{q}(t)
$$

where $B_{1}, B_{2}$, and $B_{3} \in \mathbb{R}$ are unknown positive constants.

The torque produced about the knee is controlled through muscle forces that are elicited by NMES. For simplicity (and without loss of generality), the development in this paper focuses on producing knee torque through muscle tendon forces, denoted by $F_{T}(t) \in \mathbb{R}$, generated by electrical stimulation of the quadriceps (i.e., we do not consider antagonistic muscle forces). The knee torque is related to the muscle tendon force as

$$
\tau(t)=\zeta(q(t)) F_{T}(t)
$$

where $\zeta(q(t)) \in \mathbb{R}$ denotes a positive moment arm that changes with the extension and flexion of the leg as shown in [18] and [19]. As indicated in [18] and [20] the moment arm has unique values for a given range of motion, while in [19], the moment arm's unique values are obtained for the entire range of motion and can be considered as a continuously differentiable, positive, monotonic, and bounded function with a bounded first time derivative. The tendon force $F_{T}(t)$ in (4) is defined as

$$
F_{T}=F \cos a(q)
$$

where $a(q(t))$ is defined as the pennation angle between the tendon and the muscle. The pennation angle of human quadriceps muscle changes monotonically during quadriceps contraction and is a continuously differentiable, positive, monotonic, and bounded function with a bounded first time derivative [21]. The relationship between muscle force and applied voltage is denoted by the unknown function $\eta(t) \in \mathbb{R}$ as

$$
F(t)=\eta(t) V(t)
$$

where $V(t) \in \mathbb{R}$ is the voltage applied to the quadriceps muscle by electrical stimulation. While exact force versus voltage models are debatable and contain parametric uncertainty, the generally accepted empirical relationship between the applied voltage (or similarly, current, frequency [22], [23], or pulse width) is well established. The empirical data in [22] and [23] 
indicates the function $\eta(t)$ is a continuously differentiable, nonzero, positive, monotonic, and bounded function, and its first time derivative is bounded.

The total force generated at the tendon could be considered as the sum of forces generated by an active element (often denoted by $F_{C E}$ ), the tension generated by a passive elastic element (often denoted by $F_{P E}$ ), and forces generated by viscous fluids (often denoted by $F_{V E}$ ). These forces have dynamic characteristics. For example, the passive element increases with increasing muscle length, and the muscle stiffness has been reported to change by greater than two orders of magnitude [24] under dynamic contractions. The muscle model in the paper considers the total muscle force composed of the sum of these elements as the function of an unknown nonlinear function $\eta(t)$ and an applied voltage $V(t)$. The introduction of the unknown nonlinear function $\eta(t)$ enables the muscle contraction to be considered under general dynamic conditions in the subsequent control development. Expressing the muscle contraction forces in this manner enables the development of a control method that is robust to changes in the forces, because these effects are included in the uncertain nonlinear muscle model that is incorporated in the stability analysis.

\section{CONTROl DeVElopment}

A high-level objective of NMES is to enable a person to achieve some functional task [i.e., functional electrical stimulation (FES)]. Towards this goal, the objective of the current effort is to develop a NMES controller to produce a knee position trajectory that will enable a human shank to track a desired trajectory, denoted by $q_{d}(t) \in \mathbb{R}$. The desired trajectory can be any continuous signal (or a simple constant setpoint). In the subsequent experimental results, the desired trajectories were selected as periodic signals (for simplicity and without loss of generality) of different frequencies and step functions with changes in the dynamic load. Although such trajectories may not be truly functional, trajectory-based movements are necessary for the performance of many FES augmented tasks (e.g., repetitive stepping during walking). Whether the desired trajectories are based on limb position, as in the current result, or other information (e.g., desired joint kinetics or kinematics), the ability to precisely track a desired pattern is fundamental to eliciting reproducible movement patterns during functional tasks.

To quantify the objective, a position tracking error, denoted by $e_{1}(t) \in \mathbb{R}$, is defined as

$$
e_{1}(t)=q_{d}(t)-q(t)
$$

where $q_{d}(t)$ is an a priori trajectory which is designed such that $q_{d}(t), q_{d}^{i}(t) \in \mathcal{L}_{\infty}$, where $q_{d}^{i}(t)$ denotes the $i$ th derivative for $i=1,2,3,4$. To facilitate the subsequent analysis, filtered tracking errors, denoted by $e_{2}(t)$ and $r(t) \in \mathbb{R}$, are defined as

$$
\begin{aligned}
e_{2}(t) & =\dot{e}_{1}(t)+\alpha_{1} e_{1}(t) \\
r(t) & =\dot{e}_{2}(t)+\alpha_{2} e_{2}(t)
\end{aligned}
$$

where $\alpha_{1}, \alpha_{2} \in \mathbb{R}$ denote positive constants. The filtered tracking error $r(t)$ is introduced to facilitate the closed-loop error system development and stability analysis but is not used in the controller because of a dependence on acceleration measurements.

After multiplying (9) by $J$ and utilizing the expressions in (1) and (4)-(8), the following expression can be obtained:

$$
J r=W-\Omega V+\tau_{d}
$$

where $W\left(\dot{e}_{1}, e_{2}, t\right) \in \mathbb{R}$ is an auxiliary signal defined as

$$
W=J\left(\ddot{q}_{d}+\alpha_{1} \dot{e}_{1}+\alpha_{2} e_{2}\right)+M_{e}+M_{g}+M_{v}
$$

and the continuous, positive, monotonic, and bounded auxiliary function $\Omega(q, t) \in \mathbb{R}$ is defined as

$$
\Omega=\zeta \eta \cos a .
$$

After multiplying (10) by $\Omega^{-1}(q, t) \in \mathbb{R}$, the following expression is obtained:

$$
J_{\Omega} r=W_{\Omega}-V+\tau_{d \Omega}
$$

where $J_{\Omega}(q, t) \in \mathbb{R}, \tau_{d \Omega}(q, t) \in \mathbb{R}$, and $W_{\Omega}\left(\dot{e}_{1}, e_{2}, t\right) \in \mathbb{R}$ are defined as

$$
\begin{aligned}
J_{\Omega} & =\Omega^{-1} J, \quad \tau_{d \Omega}=\Omega^{-1} \tau_{d} \\
W_{\Omega} & =\Omega^{-1} W=J_{\Omega}\left(\ddot{q}_{d}+\alpha_{1} \dot{e}_{1}+\alpha_{2} e_{2}\right)+M_{e_{\Omega}}+M_{g_{\Omega}}+M_{v_{\Omega}} .
\end{aligned}
$$

To facilitate the subsequent stability analysis, the open-loop error system for (13) can be determined as

$$
J_{\Omega} \dot{r}=-\frac{1}{2} \dot{J}_{\Omega} r+N-\dot{V}-e_{2}
$$

where $N\left(e_{1}, e_{2}, r, t\right) \in \mathbb{R}$ denotes the unmeasurable auxiliary term

$$
N=\dot{W}_{\Omega}+e_{2}-\frac{1}{2} \dot{J}_{\Omega} r+\dot{\tau}_{d \Omega}(q, t) .
$$

To further facilitate the analysis, another unmeasurable auxiliary term, $N_{d}\left(q_{d}, \dot{q}_{d}, \ddot{q}_{d}, \dddot{q}_{d}, t\right) \in \mathbb{R}$, is defined as

$$
\begin{aligned}
N_{d}=\dot{J}_{\Omega}\left(q_{d}\right) \ddot{q}_{d}+ & J_{\Omega}\left(q_{d}\right) \dddot{q}_{d}+\dot{M}_{e_{\Omega}}\left(q_{d}\right) \\
& +\dot{M}_{g_{\Omega}}\left(q_{d}\right)+\dot{M}_{v_{\Omega}}\left(q_{d}\right)+\dot{\tau}_{d_{\Omega}}\left(q_{d}, t\right) .
\end{aligned}
$$

After adding and subtracting (16) to (14), the open-loop error system can be expressed as

$$
J_{\Omega} \dot{r}=-\dot{V}-e_{2}+\tilde{N}+N_{d}-\frac{1}{2} \dot{J}_{\Omega} r
$$

where the unmeasurable auxiliary term $\tilde{N}\left(e_{1}, e_{2}, r, t\right) \in \mathbb{R}$ is defined as

$$
\tilde{N}(t)=N-N_{d}
$$

Motivation for expressing the open-loop error system as in (17) is given by the desire to segregate the uncertain nonlinearities and disturbances from the model into terms that are bounded by state-dependent bounds and terms that are upper bounded by constants. Specifically, the mean value theorem can be applied to upper bound $\tilde{N}\left(e_{1}, e_{2}, r, t\right)$ by state-dependent terms as

$$
\|\tilde{N}\| \leq \rho(\|z\|)\|z\|
$$


where $z(t) \in \mathbb{R}^{3}$ is defined as

$$
z(t) \triangleq\left[\begin{array}{lll}
e_{1}^{T} & e_{2}^{T} & r^{T}
\end{array}\right]^{T}
$$

and the bounding function $\rho(\|z\|)$ is a positive, globally invertible, nondecreasing function. The fact that $q_{d}(t)$, $q_{d}^{i}(t) \in \mathcal{L}_{\infty} \forall i=1,2,3,4$ can be used to upper bound $N_{d}\left(q_{d}, \dot{q}_{d}, \ddot{q}_{d}, \dddot{q}_{d}, t\right)$ as

$$
\left\|N_{d}\right\| \leq \zeta_{N_{d}} \quad\left\|\dot{N}_{d}\right\| \leq \zeta_{\dot{N}_{d}}
$$

where $\zeta_{N_{d}}$ and $\zeta_{\dot{N}_{d}} \in \mathbb{R}$ are known positive constants.

Based on the dynamics given in (1)-(6), the RISE-based voltage control input $V(t)$ is designed as

$$
\begin{aligned}
V(t) \triangleq\left(k_{s}\right. & +1) e_{2}(t)-\left(k_{s}+1\right) e_{2}(0) \\
& +\int_{0}^{t}\left[\left(k_{s}+1\right) \alpha_{2} e_{2}(\tau)+\beta \operatorname{sgn}\left(e_{2}(\tau)\right)\right] d \tau
\end{aligned}
$$

where $k_{s}, \beta \in \mathbb{R}$ denote positive constant adjustable control gains, and $\operatorname{sgn}(\cdot)$ denotes the signum function. Although the control input is present in the open-loop error system in (10), an extra derivative is used to develop the open-loop error system in (17) to facilitate the design of the RISE-based controller. Specifically, the time-derivative of the RISE input in (22) looks like a discontinuous sliding mode controller. Sliding mode control is desirable because it is a method that can be used to reject the additive bounded disturbances present in the muscle dynamics (e.g., muscle spasticity, load changes, electromechanical delays) while still obtaining an asymptotic stability result. The disadvantage of a sliding mode controller is that it is discontinuous. By structuring the open-loop error system as in (17), the RISE controller in (22) can be implemented as a continuous controller (i.e., the unique integral of the sign of the error) and still yield an asymptotic stability result (see Theorem 1 and the stability proof in the Appendix). Without loss of generality, the developed voltage control input can be implemented through various modulation methods (i.e., voltage, frequency, or pulse width modulation).

\section{STABILITY ANALYSIS}

Theorem 1: The controller given in (22) ensures that all system signals are bounded under closed-loop operation. The position tracking error is regulated in the sense that

$$
\left\|e_{1}(t)\right\| \rightarrow 0 \quad \text { as } t \rightarrow \infty
$$

and the controller yields semi-global asymptotic tracking provided the control gain $k_{s}$, introduced in (22) is selected sufficiently large, and $\beta$ is selected according to the following sufficient condition:

$$
\beta>\left(\zeta_{N_{d}}+\frac{1}{\alpha_{2}} \zeta_{\dot{N}_{d}}\right)
$$

where $\zeta_{N_{d}}$ and $\zeta_{\dot{N}_{d}}$ are introduced in (21).
Proof: See the Appendix.

\section{EXPERIMENTAL RESULTS}

Experiments were performed using the RISE controller given in (22). The voltage controller was implemented through an amplitude modulation scheme composed of a variable amplitude positive square wave with a fixed pulse width of $100 \mu \mathrm{s}$ and fixed frequency of $30 \mathrm{~Hz}$. The $100 \mu$ s pulse width and the $30 \mathrm{~Hz}$ stimulation frequency were chosen a priori and represent parametric settings that are within the ranges typically reported during NMES studies. During stimulation at $100 \mu$ s pulse widths, human skeletal muscle response to changes in stimulation amplitude (force-amplitude relationship) and frequency (force-frequency relationship) are highly predictable and thus deemed appropriate for use in the present study. The $30 \mathrm{~Hz}$ stimulation was selected based on force-frequency curves [25] which show that as stimulation frequency is increased muscle force increases to a saturation limit. Higher frequencies can be chosen to generate more force up to a saturation limit, but muscles tend to fatigue faster at higher frequencies. The $30 \mathrm{~Hz}$ pulse wave yields reduced fatigue in comparison to higher frequencies but lower frequencies tend to produce rippled knee motion [25], [26]. Therefore stimulation frequencies in the range of $30-40 \mathrm{~Hz}$ is an optimal choice for conducting external electrical stimulation. The following results indicate that the RISE algorithm was able to minimize the knee angle error while dynamically tracking a desired trajectory.

\section{A. Testbed and Protocol}

The testbed consists of a custom computer controlled stimulation circuit and a modified leg extension machine (LEM). The LEM was modified to include optical encoders. The LEM allows seating adjustments to ensure the rotation of the knee is about the encoder axis. A $4.5 \mathrm{~kg}(10 \mathrm{lb})$ load was attached to the weight bar of the LEM, and a mechanical stop was used to prevent hyperextension.

In the experiment, bipolar self-adhesive neuromuscular stimulation electrodes were placed over the distal-medial and proximal-lateral portion of the quadriceps femoris muscle group and connected to the custom stimulation circuitry. Prior to participating in the study, written informed consent was obtained from all the subjects, as approved by the Institutional Review Board at the University of Florida. Tracking experiments for a two period desired trajectory were conducted on both legs of five subjects. The subjects included two healthy females and three healthy males in the age group of 22-26 years. The electrical stimulation responses of healthy subjects have been reported as similar to paraplegic subjects' responses [27]-[30]. Therefore, healthy subjects were used in NMES experiments as substitute for paraplegic patients which were not available. As described in Section V-B, the results were approximately equal across the subjects (i.e., a standard deviation of $0.53^{\circ}$ of root mean squared (rms) tracking error). Therefore, additional experiments were conducted on a single subject's leg to illustrate the applicability of the controller for different conditions. 
TABLE I

TABulated Results Indicate That the Test SubJect Was not LEARNING THE DESIRED TRAJECTORY SinCE THE RMS ERRORS ARE RELATIVELY EQUAL FOR EACH TRIAL

\begin{tabular}{|c|c|}
\hline Trial & RMS error (in deg.) \\
\hline 1 & 4.35 \\
\hline 2 & 4.28 \\
\hline 3 & 4.26 \\
\hline 4 & 4.29 \\
\hline
\end{tabular}

During the experiments each subject was instructed to relax and to allow the stimulation to control the limb motion (i.e., the subjects were not supposed to influence the leg motion voluntarily and were not allowed to observe the desired trajectory). Varying the time period and range of motion may also help to reduce any possible trajectory learning and anticipation by a healthy subject. To experimentally examine if any trajectory learning occurred, four successive tests were conducted on a healthy subject with a two minute interval between trials. The experiments were conducted for $15 \mathrm{~s}$ on a dual period trajectory of 4 and $6 \mathrm{~s}$. The resulting rms errors are given in Table I. The results in Table I illustrate that trajectory learning by the subject is not apparent since the standard deviation between the successive trials is $0.039^{\circ}$.

\section{B. Results and Discussion}

The experimental results of five subjects tested for the two period desired trajectory depicted in Fig. 2, are summarized in Table II. In Table II, the maximum steady-state error is defined as the maximum absolute value of error that occurs after $4 \mathrm{~s}$ of the trial. The maximum steady-state errors range from $4.25^{\circ}$ to $7.55^{\circ}$ with a mean of $6.32^{\circ}$ and a standard deviation of $1.18^{\circ}$. The rms tracking errors range from $2^{\circ}$ to $3.47^{\circ}$ with a mean rms error of $2.75^{\circ}$ and a standard deviation of $0.53^{\circ}$. The tracking error results for Subject B and the corresponding output voltages computed by the RISE method (prior to voltage modulation) are shown in Fig. 2. The results successfully illustrate the ability of the RISE controller to track the desired two period trajectory.

To further illustrate the performance of the developed controller, experiments were also conducted for trajectories with faster and slower periods and larger ranges of motion. Specifically, the controller's performance was tested for a desired trajectory with a constant $2 \mathrm{~s}$ period, a constant $6 \mathrm{~s}$ period, a triple periodic trajectory with cycles of 2, 4, and $6 \mathrm{~s}$ and for a higher range of motion of $65^{\circ}$. As indicated in Table I, the results for the two period trajectory yielded similar results for all the subjects. Hence, these additional tests were performed on a single individual to simply illustrate the capabilities of the controller, with the understanding that some variations would be apparent when implemented on different individuals. The rms tracking errors and maximum steady-state errors are provided in Table III. The rms error and maximum steady state error is the lowest for a constant $6 \mathrm{~s}$ period desired trajectory and higher for faster trajectories and higher range of motion. These results are an expected outcome since tracking more aggressive trajectories generally yield more error. The triple periodic trajectory consists of a mix of slower and faster period trajectories, therefore the rms and maximum steady state error is moderate compared to the more
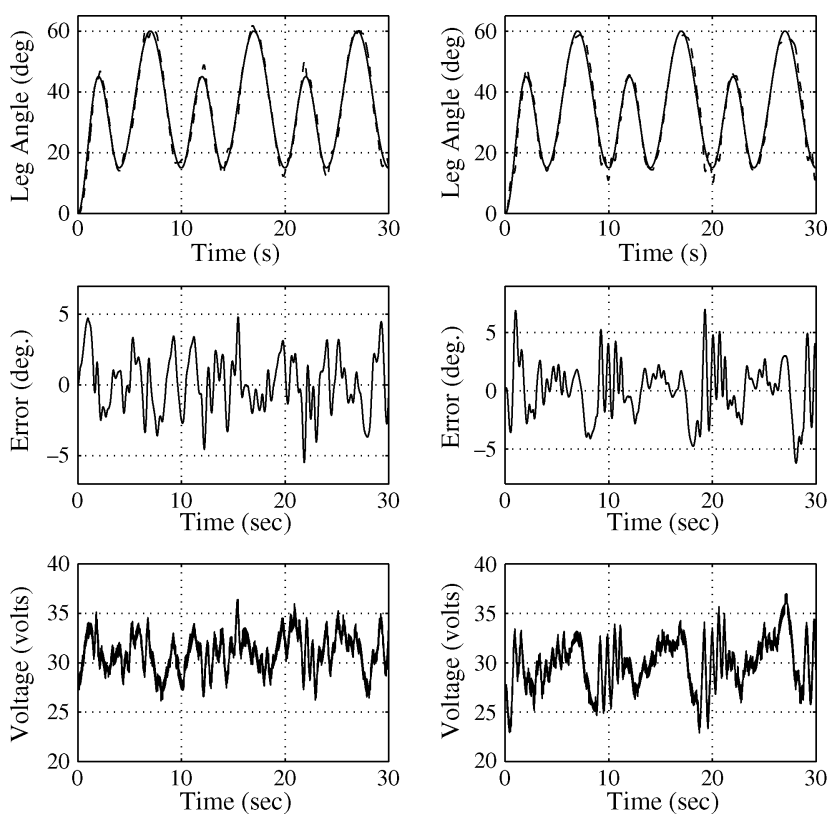

Fig. 2. Top plots: Actual left limb trajectory of a subject (solid line) versus the desired two periodic trajectory (dashed line) input. (left leg-top left plot and right leg-top right plot). Middle plots: The tracking error (desired angle minus actual angle) of a subject's leg tracking a two periodic desired trajectory. (left leg-middle left plot and right leg-middle right plot). Bottom plots: The computed RISE voltage during knee joint tracking for the case of two period trajectory (left leg_-bottom left plot and right leg—bottom right plot).

TABLE II

EXPERIMENTAL RESULTS FOR TWO PERIOD DESIRED TRAJECTORY

\begin{tabular}{|c|l|l|l|}
\hline Subject & Leg & $\begin{array}{l}\text { RMS } \\
\text { Error }\end{array}$ & $\begin{array}{l}\text { Max. } \\
\text { Steady } \\
\text { State Error }\end{array}$ \\
\hline A & Left & $2.89^{\circ}$ & $7.55^{\circ}$ \\
\hline A & Right & $2.36^{\circ}$ & $7.14^{\circ}$ \\
\hline B & Left & $2.00^{\circ}$ & $5.40^{\circ}$ \\
\hline B & Right & $2.35^{\circ}$ & $6.99^{\circ}$ \\
\hline C & Left & $2.07^{\circ}$ & $4.25^{\circ}$ \\
\hline C & Right & $2.94^{\circ}$ & $4.51^{\circ}$ \\
\hline D & Left & $3.47^{\circ}$ & $7.30^{\circ}$ \\
\hline D & Right & $2.89^{\circ}$ & $6.94^{\circ}$ \\
\hline E & Left & $3.11^{\circ}$ & $6.80^{\circ}$ \\
\hline E & Right & $3.45^{\circ}$ & $6.30^{\circ}$ \\
\hline Mean & & $\mathbf{2 . 7 5}^{\circ}$ & $\mathbf{6 . 3 2}^{\circ}$ \\
\hline Std. Dev. & & $\mathbf{0 . 5 3}^{\circ}$ & $\mathbf{1 . 1 8}^{\circ}$ \\
\hline
\end{tabular}

aggressive $2 \mathrm{~s}$ period and higher range of motion desired trajectories. Figs. 3-6 depict the errors for the experiments summarized in Table III.

Additional experiments were also conducted to examine the performance of the controller in response to step changes and changing loads. Specifically, a desired trajectory of a step input was commanded with a $10 \mathrm{lb}$ load attached to the LEM. An additional $10 \mathrm{lb}$ load was added once the limb stabilized after a step down of $15^{\circ}$. The limb was again commanded to perform a step response to raise the limb back up an additional $15^{\circ}$ with the total load of $20 \mathrm{lb}$. The results are shown in Fig. 7. The steady state error was within $\pm 1^{\circ}$. A maximum error of $3^{\circ}$ was observed when the external load was added. The results give some indication of the controller's ability to adapt to changes in load and step inputs and motivate possible future case studies 
TABLE III

SUMMARIZED EXPERIMENTAL RESULTS FOR MULTIPLE, HIGHER FREQUENCIES AND Higher RANGE OF MOTION. COLUMN (A) INDICATES RMS ERROR IN Degrees, and Column (B) Indicates MaXimum Steady STate ERror IN DEGREES

\begin{tabular}{|l|c|c|}
\hline Trajectory & A & B \\
\hline Constant 6 sec. & 2.88 & 6.13 \\
\hline Constant 2 sec. & 4.11 & 10.67 \\
\hline Triple periodic $(6,4,2)$ sec. & 3.27 & 7.82 \\
\hline $\begin{array}{l}\text { Triple periodic }(6,4,2) \text { sec with higher range } \\
\text { of motion }\end{array}$ & 5.46 & 12.48 \\
\hline
\end{tabular}
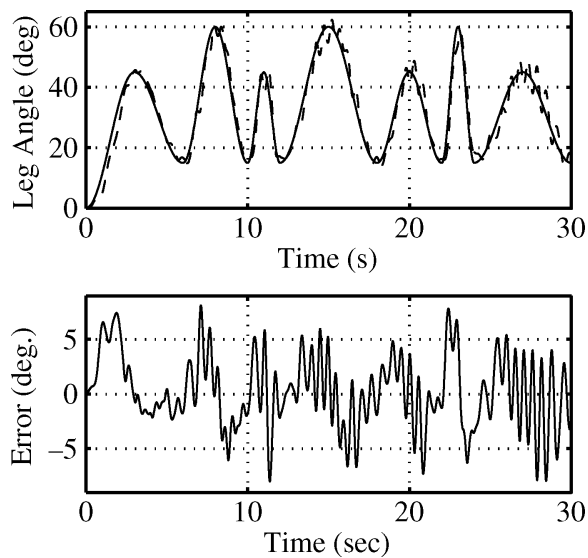

Fig. 3. Top plot: Actual limb trajectory (solid line) versus the desired triple periodic trajectory (dashed line). Bottom plot: The limb tracking error (desired angle minus actual angle) of a subject tracking a triple periodic desired trajectory.
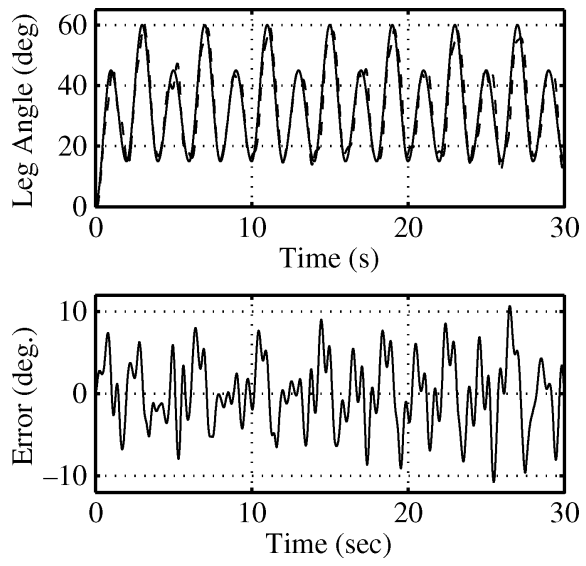

Fig. 4. Top plot: Actual limb trajectory (solid line) versus the desired constant period ( $2 \mathrm{~s}$ ) trajectory (dashed line). Bottom plot: The limb tracking error (desired angle minus actual angle) of a subject tracking a constant period ( $2 \mathrm{~s})$ desired trajectory.

with neurologically impaired individuals that express muscle spasticity.

For each experiment, the computed voltage input was modulated by a fixed pulse width of $100 \mu \mathrm{s}$ and fixed frequency of $30 \mathrm{~Hz}$. The stimulation frequency was selected based on subject comfort and to minimize fatigue. During preliminary experiments with stimulation frequencies of $100 \mathrm{~Hz}$, the subjects fatigued approximately two times faster than in the current results. The results also indicate that a $100 \mu$ s pulse width was acceptable, though future studies will investigate higher pulse
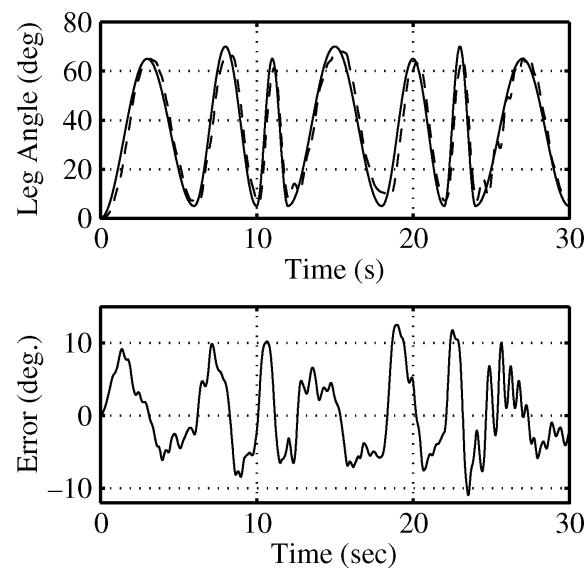

Fig. 5. Top plot: Actual limb trajectory (solid line) versus the triple periodic desired trajectory with higher range of motion (dashed line). Bottom plot: The limb tracking error (desired angle minus actual angle) of a subject tracking a triple periodic desired trajectory with higher range of motion.
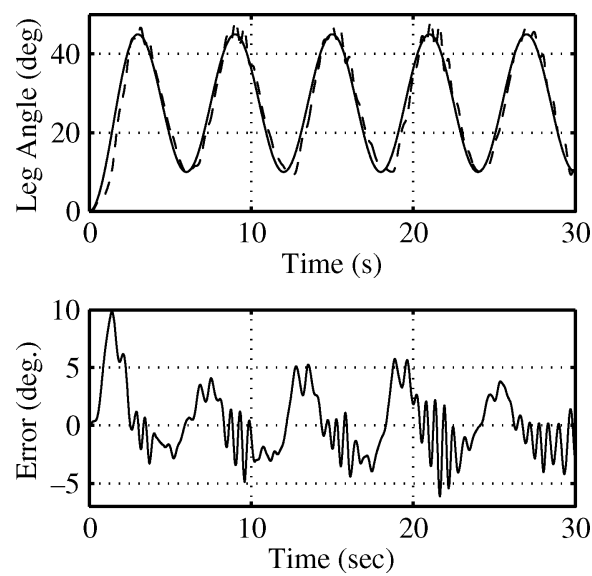

Fig. 6. Top plot: Actual limb trajectory (solid line) versus the desired constant period $(6 \mathrm{~s})$ trajectory (dashed line). Bottom plot: The limb tracking error (desired angle minus actual angle) of a subject tracking a constant period (6 s) desired trajectory.
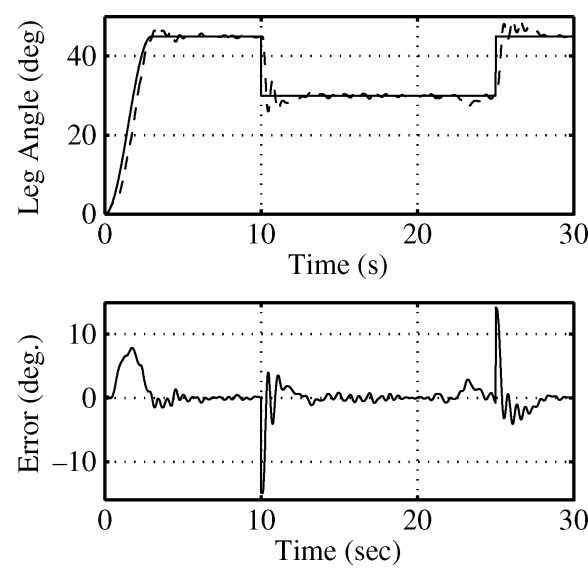

Fig. 7. Top plot : Actual limb trajectory (solid line) versus desired step trajectory (dashed line). The limb is tested for two step inputs. The load is added once the limb stabilizes ( between 23 and $24 \mathrm{~s}$ interval). Bottom plot: The limb tracking error for step inputs.

widths in the range of 300-350 $\mu$ s which recruit more slow fatiguing motor units [25]. Our previous preliminary experiments 
indicated that longer pulse widths (e.g., $1 \mathrm{~ms}$ ) produced similar effects as a direct current voltage.

The use of the RISE control structure is motivated by its implicit learning characteristics [31] and its ability to compensate for additive system disturbances and parametric uncertainties in the system. The advantage of the RISE controller is that it does not require muscle model knowledge and guarantees asymptotic stability of the nonlinear system. The experimental results indicate that this feedback method may have promise in some clinical applications.

Although the RISE controller was successfully implemented, the performance of the controller may be improved by including a feedforward control structure such as neural networks (a black box function approximation technique) or physiological/phenomenological muscle models. Since the RISE controller is a high gain feedback controller that yields asymptotic performance, adding a feedforward control element may improve transient and steady state performance and reduce the overall control effort, thereby reducing muscle fatigue. Another possible improvement to the controller is to account for fatigue. Fatigue can be reduced for short durations by selecting optimal stimulation parameters, but FES may require a controller that adapts with fatigue to yield performance gains for longer time durations. Therefore, our future goal will be to include a fatigue model in the system to enhance the controller performance.

\section{CONCLUSION}

A Lyapunov-based stability analysis indicates that the developed closed-loop nonlinear control method yields asymptotic tracking for a nonlinear muscle activation and limb dynamics, even in the presence of additive disturbances. Experiments using external electrodes on human subjects demonstrated the ability of the RISE controller to enable a limb to track a desired trajectory composed of varying amplitude and frequency sinusoids, step changes, and changes in the load. Specifically, the experimental results indicated that with no muscle model (and only voltage amplitude modulation), the RISE algorithm could determine the appropriate stimulation voltage for the tracking objective. For the fastest tested trajectory the maximum steady-state tracking errors were approximately $10^{\circ}$, whereas the maximum steady-state error in slower trajectories were as little as approximately $4^{\circ}$. An advantage of this controller is that it can be applied without knowledge of patient specific parameters like limb mass or inertia, limb center of gravity location, parameters that model passive, and elastic force elements. Thus, its application would not require specific expertise or extensive testing prior to use. The control development also accounts for unmodeled disturbance (e.g., muscle spasticity) that are commonly observed in clinical populations. The proposed strategy holds promise for clinical implementation of the controller as a therapeutic tool to enhance muscle function during isolated joint movements. We do, however, recognize that our results have yet to be demonstrated to elicit functional movements (e.g., walking) in populations without the ability to voluntarily activate their muscles.
As such, future directions will focus on such studies to demonstrate the effectiveness of the controller under such conditions. Although the trajectories used in the experiments may not be truly functional, the controller can be applied to any continuous trajectory. This is clinically relevant because trajectory-based movements are necessary for the performance of many FES augmented tasks (e.g., repetitive stepping during walking). Whether the desired trajectories are based on limb position, as in the current result, or other information (e.g., desired joint kinetics or kinematics), the ability to precisely track a desired pattern is fundamental to eliciting reproducible movement patterns during functional tasks. An advantage of the control development is that it allows for inter- as well as intra-individual variations in trajectory tracking (i.e. task performance) to be accounted for both within and between sessions (e.g., during rehabilitation training), thus potentially providing a tool to aid in the future advancement of rehabilitation. A possible disadvantage of the controller is that high gains are used to achieve the robustness to disturbances and unmodeled effects. Further efforts will investigate augmenting the developed RISE structure with feedforward control architectures that can accommodate for disturbances without requiring high gain feedback.

\section{APPENDIX}

The following development is a brief Lyapunov-based proof. The complete stability analysis and development of the RISE method can be found in [15], [16], [32], [33].

Proof for theorem 1: Let $\mathcal{D} \subset \mathbb{R}^{3+1}$ be a domain containing $y(t)=0$, where $y(t) \in \mathbb{R}^{3+1}$ is defined as

$$
y(t) \triangleq\left[\begin{array}{ll}
z^{T} & \sqrt{P(t)}
\end{array}\right]^{T}
$$

and the auxiliary function $P(t) \in \mathbb{R}$ is defined as

$$
P(t) \triangleq \beta\left\|e_{2}(0)\right\|-e_{2}(0)^{T} N_{d}(0)-\int_{0}^{t} L(\tau) d \tau .
$$

The auxiliary function $L(t) \in \mathbb{R}$ in (26) is defined as

$$
L(t) \triangleq r^{T}\left(N_{d}(t)-\beta \operatorname{sgn}\left(e_{2}\right)\right) .
$$

The derivative of $\dot{P}(t) \in \mathbb{R}$ can be expressed as

$$
\dot{P}(t)=-L(t) \triangleq-r^{T}\left(N_{d}(t)-\beta \operatorname{sgn}\left(e_{2}\right)\right) .
$$

Provided the sufficient conditions stated in Theorem 1 are satisfied, the following inequality can be obtained:

$$
\int_{0}^{t} L(\tau) d \tau \leq \beta\left\|e_{2}(0)\right\|-e_{2}(0)^{T} N_{d}(0) .
$$

Hence, (29) can be used to conclude that $P(t) \geq 0$.

Let $V_{L}(y, t) \in \mathbb{R}$ denote a continuously differentiable, positive definite function defined as

$$
V_{L}(y, t) \triangleq e_{1}^{T} e_{1}+\frac{1}{2} e_{2}^{T} e_{2}+\frac{1}{2} r^{T} J_{\Omega} r+P
$$


which satisfy the inequalities

$$
U_{1}(y) \leq V_{L}(y, t) \leq U_{2}(y)
$$

provided the sufficient condition introduced Theorem 1 is satisfied, where $U_{1}(y), U_{2}(y) \in \mathbb{R}$ are continuous, positive definite functions. After taking the derivative of (30) and utilizing (8), (9), (17), (26), and (27) $\dot{V}_{L}(y, t)$ can be expressed as

$$
\begin{aligned}
\dot{V}_{L}(y, t)=-\left(2 \alpha_{1}-1\right) e_{1}^{T} e_{1}- & \left(\alpha_{2}-1\right) e_{2}^{T} e_{2} \\
& -r^{T} r+r^{T} \tilde{N}-k_{s} r^{T} r .
\end{aligned}
$$

As shown in (32) the unique integral signum term in the RISE controller is used to compensate for the disturbance terms included in $N_{d}\left(q_{d}, \dot{q}_{d}, \ddot{q}_{d}, \dddot{q}_{d}, t\right)$, provided the control gain $\beta$ is selected according to (24). Using (19), the term $r^{T}(t) \tilde{N}\left(e_{1}, e_{2}, r, t\right)$, can be upper bounded by following inequality:

$$
\|r \tilde{N}\| \leq \rho(\|z\|)\|z\|\|r\|
$$

to obtain

$$
\begin{aligned}
\dot{V}_{L}(y, t) \leq-\min \left\{2 \alpha_{1}-\right. & \left.1, \alpha_{2}-1,1\right\}\|z\|^{2} \\
& +\left[\rho(\|z\|)\|z\|\|r\|-k_{s}\|r\|^{2}\right] .
\end{aligned}
$$

Completing the squares for the bracketed terms in (33) yields

$$
\dot{V}_{L}(y, t) \leq-\min \left\{2 \alpha_{1}-1, \alpha_{2}-1,1\right\}\|z\|^{2}+\frac{\rho^{2}(\|z\|)\|z\|^{2}}{4 k_{s}} \text {. }
$$

The following expression can be obtained from (34):

$$
\dot{V}_{L}(y, t) \leq-U(y)
$$

where $U(y)$ is a continuous positive definite function, provided $k_{s}$ is selected sufficiently large based on the initial conditions of the system. That is, the region of attraction can be made arbitrarily large to include any initial conditions by increasing the control gain $k_{s}$ (i.e., a semi-global type of stability result). By invoking [34, Theorem 8.4], the semi-global asymptotic tracking in (23) can be achieved.

\section{REFERENCES}

[1] P. H. Peckham and D. B. Gray, "Functional neuromuscular stimulation," J. Rehab. Res. Develop., vol. 33, pp. 9-11, 1996.

[2] J. J. Abbas and H. J. Chizeck, "Feedback control of coronal plane hip angle in paraplegic subjects using functional neuromuscular stimulation," IEEE Trans. Biomed. Eng., vol. 38, no. 7, pp. 687-698, Jul. 1991.

[3] N. Lan, P. E. Crago, and H. J. Chizeck, "Control of end-point forces of a multijoint limb by functional neuromuscular stimulation," IEEE Trans. Biomed. Eng., vol. 38, no. 10, pp. 953-965, Oct. 1991.

[4] T. Schauer, N. O. Negard, F. Previdi, K. J. Hunt, M. H. Fraser, E. Ferchland, and J. Raisch, "Online identification and nonlinear control of the electrically stimulated quadriceps muscle," Control Eng. Practice, vol. 13, pp. 1207-1219, 2005.

[5] K. Stegath, N. Sharma, C. M. Gregory, and W. E. Dixon, "An extremum seeking method for non-isometric neuromuscular electrical stimulation," in IEEE Int. Conf. Syst., Man, Cybern., Oct. 2007, pp. $2528-2532$.

[6] M. Ferrarin, E. Pavan, R. Spadone, R. Cardini, and C. Frigo, "Standing up exerciser based on functional electrical stimulation and body weight relief," Med. Biol. Eng. Comput., vol. 40, no. 3, pp. 282-289, 2002.

[7] G. Khang and F. E. Zajac, "Paraplegic standing controlled by functional neuromuscular stimulation: Part I-Computer model and control-system design," IEEE Trans. Biomed. Eng., vol. 36, no. 9, pp. 873-884, Sep. 1989.
[8] F. Previdi, M. Ferrarin, S. Savaresi, and S. Bittanti, "Gain scheduling control of functional electrical stimulation for assisted standing up and sitting down in paraplegia: a simulation study," Int. J. Adapt. Control, vol. 19 , pp. 327-338, 2005.

[9] H. Kordylewski and D. Graupe, "Control of neuromuscular stimulation for ambulation by complete paraplegics via artificial neural networks," Neurol. Res., vol. 23, no. 5, pp. 472-481, 2001.

[10] J. A. Riess and J. J. Abbas, "Adaptive neural network control of cyclic movements using functional neuromuscular stimulation," IEEE Trans. Rehabil. Eng., vol. 8, no. 1, pp. 42-52, Mar. 2000

[11] K. Y. Tong and M. H. Granat, "Gait-control system for functional electrical stimulation using neural networks," Med. Bio. Eng. Comput., vol. 37, pp. 35-41, 1999.

[12] F. Sepulveda, Computer Techniques in Medical and biotechnology Systems. Norwell, MA: Kluwer, 2003.

[13] J. P. Giuffrida and P. E. Crago, "Functional restoration of elbow extension after spinal-cord injury using a neural network-based synergistic FES controller," IEEE Trans. Neural Syst. Rehabil. Eng., vol. 13, no. 2, pp. 147-152, Jun. 2005.

[14] K. Stegath, N. Sharma, C. Gregory, and W. Dixon, "Nonlinear tracking control of a human limb via neuromuscular electrical stimulation," in American Control Conference, Seattle, WA, Jun. 2008, pp. 1941-1946.

[15] P. M. Patre, W. MacKunis, C. Makkar, and W. E. Dixon, "Asymptotic tracking for systems with structured and unstructured uncertainties," IEEE Trans. Control. Syst. Tech., vol. 16, no. 2, pp. 373-379, Mar. 2008.

[16] P. M. Patre, W. MacKunis, C. Makkar, and W. E. Dixon, "Asymptotic tracking for systems with structured and unstructured uncertainties," in IEEE Conf. Decision Control , 2006, pp. 441-446.

[17] M. Ferrarin and A. Pedotti, "The relationship between electrical stimulus and joint torque: A dynamic model," IEEE Trans. Rehabil. Eng., vol. 8, no. 3, pp. 342-352, Sep. 2000.

[18] J. L. Krevolin, M. G. Pandy, and J. C. Pearce, "Moment arm of the patellar tendon in the human knee," J. Biomech., vol. 37, pp. 785-788, 2004.

[19] W. L. Buford, Jr., F. M. Ivey, Jr., J. D. Malone, R. M. Patterson, G. L. Peare, D. K. Nguyen, and A. A. Stewart, "Muscle balance at the knee-Moment arms for the normal knee and the ACL-Minus knee," IEEE Trans. Rehabil. Eng, vol. 5, no. 4, pp. 367-379, 1997.

[20] R. Riener and T. Fuhr, "Patient-driven control of FES-supported standing up: A simulation study," IEEE Trans. Rehabil. Eng., vol. 6, no. 2, pp. 113-124, Jun. 1998.

[21] O. M. Rutherford and D. A. Jones, "Measurement of fibre pennation using ultrasound in the human quadriceps in vivo," Eur. J. Appl. Phys., vol. 65 , pp. 433-437, 1992.

[22] R. Nathan and M. Tavi, "The influence of stimulation pulse frequency on the generation of joint moments in the upper limb," IEEE Trans. Biomed. Eng., vol. 37, no. 3, pp. 317-322, Mar. 1990.

[23] T. Watanabe, R. Futami, N. Hoshimiya, and Y. Handa, "An approach to a muscle model with a stimulus frequency-force relationship for FES applications," IEEE Trans. Rehabil. Eng., vol. 7, no. 1, pp. 12-17, Mar. 1999.

[24] J. M. Yohanan Giat and M. Levy, "A musculotendon model of the fatigue profiles of paralyzed quadriceps muscle under FES," IEEE Trans. Biomed. Eng., vol. 40, no. 7, pp. 664-674, Jul. 1993.

[25] R. Riener and J. Quintern, Biomechanics and Neural Control of Posture and Movement, J. Winters and P. E. Crago, Eds. New York: SpringerVerlag, 2000.

[26] R. Riener, J. Quintern, and G. Schmidt, "Biomechanical model of the human knee evaluated by neuromuscular stimulation," J. Biomech., vol. 29, pp. 1157-1167, 1996.

[27] G.-C. Chang, L. Jer-Junn, L. Gon-Der, L. Jin-Shin, C. Cheng-Kung, K. Bor-Lin, and K. Te-Son, "A neuro-control system for the knee joint position control with quadriceps stimulation," IEEE Trans. Rehabil. Eng, vol. 5, no. 1, pp. 2-11, Mar. 1997.

[28] J. Hausdorff and W. Durfee, "Open-loop position control of the knee joint using electrical stimulation of the quadriceps and hamstrings," Med. Biol. Eng. Comput., vol. 29, pp. 269-280, 1991.

[29] S. Jezernik, R. Wassink, and T. Keller, "Sliding mode closed-loop control of FES: Controlling the shank movement," IEEE Trans. Biomed. Eng, vol. 51, no. 2, pp. 263-272, Feb. 2004.

[30] K. Kurosawa, R. Futami, T. Watanabe, and N. Hoshimiya, "Joint angle control by FES using a feedback error learning controller," IEEE Trans. Neural Syst. Rehabil. Eng., vol. 13, no. 3, pp. 359-371, Sep. 2005.

[31] Z. Qu and J. Xu, "Model-based learning controls and their comparisons using lyapunov direct method," Asian J. Control, vol. 4, no. 1 , pp. 99-110, 2002. 
[32] B. Xian, D. M. Dawson, M. S. de Queiroz, and J. Chen, "A continuous asymptotic tracking control strategy for uncertain multi-input nonlinear systems," IEEE Trans. Autom. Control, vol. 49, no. 7, pp. 1206-1211, Jul. 2004.

[33] C. Makkar, G. Hu, W. G. Sawyer, and W. E. Dixon, "Lyapunov-based tracking control in the presence of uncertain nonlinear parameterizable friction," IEEE Trans. Autom. Control, vol. 52, no. 10, pp. 1988-1994, Oct. 2007.

[34] H. Khalil, Nonlinear Systems. Englewood Cliffs, NJ: Prentice Hall, 2002.

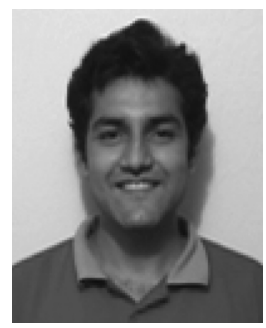

Nitin Sharma received the B.E. degree in industrial engineering from Thapar Institute of Engineering and Technology (Currently, Thapar University), Patiala, India, in 2004. He is currently working toward the Ph.D. degree in the Department of Mechanical and Aerospace Engineering, University of Florida, Gainesville.

After finishing his undergraduate studies, he worked as an Assistant Manager with Maruti Suzuki Ltd., Gurgaon, India until 2006.

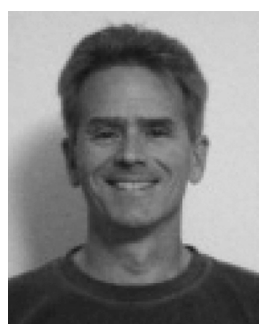

Keith Stegath received the B.S. degree in electrical engineering and the M.S. degree in mechanical engineering from the University of Florida, Gainesville, in 2005 and 2007, respectively.

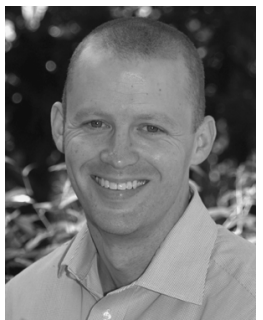

Chris M. Gregory received the Ph.D. degree in exercise physiology from the University of Georgia in 2002 and completed subsequent postdoctoral training in muscle biology at the University of Florida, in 2005.

He received a Career Development Award (level I) from the Department of Veterans Affairs in 2005 and was named to the faculty at University of Florida in 2006 as a Research Assistant Professor. His research focuses on the study of skeletal muscle form and function. Specifically, he studies the impact that skeletal muscle characteristics have on functional performance in a variety of populations that experience muscle atrophy following periods of disuse. He was recently awarded a Career Development Award (level II) from the Department of Veterans Affairs as well as a Rehabilitation Research Career Development Award (K12) from the National Institutes of Health. During his career, he has published over 80 manuscripts, conference proceedings, book chapters and abstracts on topics including muscle physiology, biochemistry, exercise and electrical stimulation of skeletal muscle.

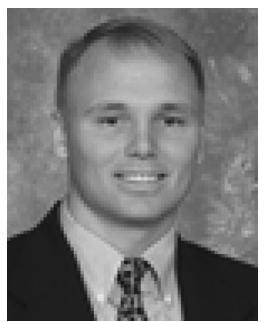

Warren E. Dixon received the Ph.D. degree from the Department of Electrical and Computer Engineering from Clemson University, Clemson, SC, in 2000 .

After completing his doctoral studies he was selected as an Eugene P. Wigner Fellow at Oak Ridge National Laboratory (ORNL). In 2004, he joined the faculty of the University of Florida in the Mechanical and Aerospace Engineering Department. His main research interest has been the development and application of Lyapunov-based control techniques for uncertain nonlinear systems. He has published two books, an edited collection, four chapters, and over 180 refereed journal and conference papers. He is an Associate Editor for Automatica, International Journal of Robust and Nonlinear Control, and Journal of Robotics.

Dr. Dixon was awarded the 2001 ORNL Early Career Award for Engineering Achievement for his contributions to Lyapunov-based control methods. He was awarded a NSF CAREER Award in 2006 for new development and application of Lyapunov-based control methods. He was also awarded the 2006 IEEE Robotics and Automation Society (RAS) Early Academic Career Award. He serves on numerous technical committees, is a member of numerous conference program committees, and serves on the conference editorial board for the IEEE CSS and RAS and the ASME DSC. He served as an appointed member to the IEEE CSS Board of Governors for 2008. He is currently an Associate Editor for IEEE TRANSACTIONS ON SYSTEMS MAN AND CYBERNETICS: PART B CYBERNETICS. 